\begin{tabular}{||l|l|}
\hline Citation & $\begin{array}{l}\text { Tuba Ayhan, Tom Redant, Marian Verhelst, and Wim Dehaene (2012) } \\
\text { Towards a fast and hardware efficient sub-mm precision ranging system } \\
\text { In Signal Processing Systems (SiPS), 2012 IEEE Workshop on, pp. 203-208.. }\end{array}$ \\
\hline Archived version & $\begin{array}{l}\text { Author manuscript: the content is identical to the content of the published } \\
\text { paper, but without the final typesetting by the publisher }\end{array}$ \\
\hline $\begin{array}{l}\text { Published version } \\
\text { http://dx.doi.org/10.1109/SiPS.2012.20 }\end{array}$ \\
\hline Journal homepage \\
huttp://ieeexplore.ieee.org/Xplore/home.jsp \\
\hline $\begin{array}{l}\text { tuba.ayhan@esat.kuleuven.be } \\
+32(0) 16321097\end{array}$ \\
\hline
\end{tabular}

(article begins on next page) 


\title{
TOWARDS A FAST AND HARDWARE EFFICIENT SUB-MM PRECISION RANGING SYSTEM
}

\author{
Tuba Ayhan ${ }^{1}$, Tom Redant ${ }^{1}$, Marian Verhelst ${ }^{1}$, Wim Dehaene $^{1,2}$ \\ ${ }^{1}$ ESAT-MICAS, KU Leuven, Kasteelpark Arenberg 10, B-3001 Leuven, Belgium \\ ${ }^{2}$ IMEC, Kapeldreef 75, B-3001 Leuven, Belgium \\ \{tuba.ayhan, tom.redant, marian.verhelst, wim.dehaene\}@esat.kuleuven.be
}

\begin{abstract}
A sub-mm ranging system, which estimates the time of flight of a RF signal between two nodes using Time of Arrival (ToA) estimation, is possible according to maximum likelihood estimator simulations and theoretical bounds on ToA estimation. In this paper we propose a frequency domain based ToA estimator for an indoor ranging system which is broken into 3 computational steps towards an efficiently implementable estimator. Performance of this hardware efficient estimator is comparable with the maximum likelihood estimator's and it is computationally efficient. Complexity of the computational steps can be traded off against eachother. Moreover, implementation-aware estimator provides high flexibility on choosing between transmitted signal energy, computational cost and precision of the ranging algorithm. In this work, a precision better than $1 \mathrm{~mm}$ is obtained for SNRs below $0 \mathrm{~dB}$, by transmitting an OFDM (Orthogonal Frequency Division Multiplexing) like signal whose duration is $9 \mu \mathrm{s}$, with a $6 \mathrm{GHz}$ bandwidth on a $60 \mathrm{GHz}$ carrier.
\end{abstract}

Index Terms - Ranging, $60 \mathrm{GHz}$, Time of Flight, Millimeter Wave

\section{INTRODUCTION}

Very precise position estimations with a high update rate in indoor environments are required in many scenarios such as doctor or patient monitoring in hospitals, search and rescue operations in natural disasters, mobile robot control and logistics in factories and warehouses [1]. Precision in order of millimeter is reported in ranging as given in [2] and the references given therein. The precision increases with the carrier frequency and the highest precision is achieved by a cross-correlation based method [3] at $24 \mathrm{GHz}$ carrier frequency. Sub-millimeter precision is possible using a higher carrier frequency, however cross-correlation based methods become practically infeasible for achieving high precision for high carrier frequencies. Therefore cross-correlation based

This project is funded by Flemish Agency for Innovation by Science and Technology (IWT) as SBO project OmniTrack. methods do not fulfill the high update rate requirements for a dynamical system. Moreover, realistic indoor applications demand the use of auto-correlation methods rather than crosscorrelation because of multipath distortions [4]. Here, we target to build an estimator for an indoor ranging system with the following properties:

- Ranging precision below $1 \mathrm{~mm}$,

- Adjustable maximum operating range beyond $15 \mathrm{~m}$,

- Hardware efficient and fast,

- Flexible for implementation distortion mitigation,

- Flexible for precision - energy - complexity trade-offs.

Here, the system id built to based on a ToA estimation technique. A multicarrier signal is chosen to be transmitted, where the carriers are orthogonal to each other, so an OFDMlike signal is obtained. An OFDM-like signal has many advantages for ranging applications due to its practicality in implementation, robustness to multipath fading and spectral efficiency, which is investigated in [5] particularly.

According to the Cramér Rao Lower Bound (CRLB) on ToA estimation precision [6], the precision improves with increasing carrier frequency and signal bandwith. This paper introduces an estimator that uses the frequency domain information of the received signal. The estimator is enhanced towards an hardware efficient implementation by separating it into steps to reduce the computational complexity and combined with a symbol synchronization step. The primary goal of this stepwise approach is to lower the amount of computations.

The ranging system is introduced in Section 2 together with the theoretical bounds on ranging precision that motivates us towards using the frequency domain information of the signal in our estimator. Derivation of the estimator is given in Section 3.1, followed by the implementation centric version in Sections 3.2 - 3.4, and the signal construction for this estimator is given in Section 3.5. The performance of the system is investigated for a single path environment with Additive White Gaussian (AWG) noise by simulations in Section 4 and finally the paper is concluded in Section 5. 


\section{SYSTEM MODEL AND LIMITATIONS}

The general setup described in this section will be used for a ranging application which aims to estimate the distance between the target and the base with $0.1 \mathrm{~mm}$ precision in less than $1 \mathrm{~ms}$, in a single path environment. The maximum range to be estimated is Rmax. An OFDM symbol built up by $\mathrm{N}$ equally spaced subcarriers is

$$
s(t)=\sum_{n=-N / 2}^{N / 2-1} A(n) e^{j n \omega_{\Delta} t},
$$

where $j=\sqrt{-1}, \omega_{\Delta}$ is the frequency spacing and $A$ is a pattern, chosen to generate an OFDM-like signal with a low peak to average power ratio (PAPR), such that $A(n) \in\{-1,1\}$. The period of one OFDM-like symbol is $T_{S Y M}$.

The signal is up-converted to the carrier frequency $\omega_{c}=$ $2 \pi f_{c}$ and transmitted at $t=0$, through the channel with Additive White Gaussian Noise. The signal is received after a certain delay denoted by $\tau$. At the receiver the signal is down converted and filtered to obtain $r(t)$, assuming receiver and transmitter are synchronized:

$$
r(t)=s(t-\tau) \exp \left(-j \omega_{c} \tau\right)+v(t),
$$

where $v(t)$ is Additive White Gaussian noise with a two-sided power spectral density of $N_{0} / 2$.

The distance $d$ between the target and the base, transmitter and the receiver respectively, is

$$
d=c \tau,
$$

where $c=3.10^{8} \mathrm{~m} / \mathrm{s}$ is the speed of light. Assuming the signal is transmitted at $t=0$, time of arrival is equal to the time of flight. Then, ToA ranging can be done by estimating $\tau$.

The lower bound on the standard deviation for the estimate of an unknown parameter $(\hat{\tau})$ from an observable variable is given by CRLB [4].

$$
\operatorname{var}\{\hat{\tau}\} \geq \frac{1}{\frac{E}{N_{0} / 2} \beta^{2}},
$$

where $E$ is the energy of the received signal and $\beta^{2}$ is the Effective bandwidth and given by

$$
\beta^{2}=\frac{\int_{-\infty}^{\infty}(2 \pi f)^{2}|S(f)|^{2} \mathrm{~d} f}{\int_{-\infty}^{\infty}|S(f)|^{2} \mathrm{~d} f}
$$

where $S(f)$ is the power spectral density of the signal.

The estimation precision in this paper is defined as the standard deviation on ToA estimation error $(\sigma)$ is given by

$$
\sigma=\sqrt{\mathrm{E}\left[(|\tau-\hat{\tau}|)^{2}\right]},
$$

where $\mathrm{E}[$.$] is the expectation operator and the ^symbol indi-$ cates the variables to be estimated. Mean of estimation error is not investigated since the mean of the AWG noise is zero.

Effective bandwidth of an OFDM signal approaches its passband bandwidth, $B$ (difference between its upper and lower cutoff frequencies) as the number of subcarriers increases. For a sufficient number of subcarriers, the CRLB can be rewritten as in [6]:

$$
\sigma^{2} \geq \frac{3}{4 \pi^{2} T \operatorname{SNR}\left(\left(f_{c}+\frac{B}{2}\right)^{3}-\left(f_{c}-\frac{B}{2}\right)^{3}\right)},
$$

where $T$ is the duration of the signal to be used in the estimation algorithm and SNR is the signal to noise power ratio: $E /\left(N_{0} / 2\right)$. The CRLB given in (7) is tight to the maximum likelihood estimator in the region of our interest [4].

\section{RANGING ALGORITHM}

As seen from the CRLB in (7), the bandwidth and the carrier frequency of the signal are dominating the lower limit on the estimation precision. The phase of a higher carrier frequency changes to a greater extend compared to a lower one within the same flight time, so a higher resolution is achieved using high frequencies. On the other hand, capturing that change in the phase of carrier requires super fine correlation steps or extremely high sampling rates. Alternatively, the estimator proposed in this paper extracts time of arrival information in the frequency domain to avoid the high computational cost of correlation based methods. In this section, the estimator is proposed and it is adapted towards an implementable system. This implementation-aware estimator is capable of achieving the CRLB using a $6 \mathrm{GHz}$ bandwidth signal in the unlicensed $60 \mathrm{GHz}$ ISM (Industrial, Scientific and Medical) band under the conditions that are also derived in this section.

\subsection{Estimator}

In order to build the estimator, the representation of $\tau$ in frequency domain is derived under ideal conditions for a single path without noise $\left(N_{0}=0\right)$. Given the frequency of the $n$th subcarrier is $\omega_{n}=n \omega_{\Delta}$, the phase of the $n$th subcarrier in the received signal is

$$
\varphi_{n}^{R X}=-\omega_{n} \tau-\omega_{c} \tau+\varphi_{n}^{T X}
$$

where $\varphi_{n}^{T X}$ is the phase of the subcarrier in the transmitted signal and $\varphi_{n}^{T X}=\{0, \pi\}$ since $A(n)$ is either 1 or $-1 . \varphi_{n}^{T X}$ is not an unknown and can be subtracted; so for clarity these terms are removed from the equations below without loss of generality.

The received signal is sampled with a sampling frequency $f_{s}$ that ensures Nyquist sampling criterion such that $r[l]=$ $r\left(l / f_{s}\right), l \in \mathbb{Z}^{+}$. Phase of $n$th subcarrier, calculated by DFT (Discreet Fourier Transform) of one period, is given as

$$
\rho_{n}=\left(\varphi_{n}^{R X}\right)_{\bmod 2 \pi}=-\omega_{n}^{p} \tau+2 k_{n} \pi, \quad k_{n} \in \mathbb{Z} .
$$


The passband frequency of $n$th subcarrier is $\omega_{n}^{p}=\omega_{n}+\omega_{c}$ and $\omega_{n}^{p}>\omega_{n-1}^{p}$. The matrix representation is given in (10) and (11).

$$
\begin{aligned}
& P=-W \tau+2 \pi K \\
& P=A X
\end{aligned}
$$

where $X=\left[\begin{array}{lllll}k_{-N / 2} & k_{-N / 2+1} & \ldots & k_{N / 2-1} & \tau\end{array}\right]^{\prime}, W=$ $\left[\omega_{-N / 2}^{p} \omega_{-N / 2+1}^{p} \cdots \omega_{N / 2-1}^{p}\right]^{\prime}$ and 'indicates the transpose operation. $A_{(N \times N+1)}$ is given as

$$
\begin{aligned}
A & =\left[A 1_{(N \times N)} A 2_{(N \times 1)}\right] \\
A 1 & =\operatorname{diag}(N), A 1(i, i)=2 \pi \\
A 2 & =-W
\end{aligned}
$$

where $\operatorname{diag}(N)$ denotes a diagonal matrix sized $(N \times N)$. The phase of a subcarrier $n$ cannot wrap more than the phase of subcarrier $n+1$; also the ratio between $\omega_{n}^{p}$ and $\omega_{n+1}^{p}$ determines the ratio between $k_{n}$ and $k_{n+1}$ as stated in (15) and (16).

$$
\begin{aligned}
k_{n+1}-k_{n} & \geq 0 \\
c_{n} k_{n}-k_{n+1} & \geq 0, \quad c_{n}=\frac{\omega_{n+1}^{p}}{\omega_{n}^{p}}
\end{aligned}
$$

Upper bounds for $k_{n}$ and $\tau$ are dependent on the maximum range that the solution is unique in. Lower bounds on $k_{n}$ and $\tau$ are zero, since the delay cannot be negative.

$$
\begin{array}{r}
0 \leq \tau<T_{S Y M} \\
0 \leq k_{n}<\frac{T_{S Y M} \boldsymbol{\omega}_{n}}{2 \pi} .
\end{array}
$$

In the presence of noise $\left(N_{0} \neq 0\right), X$ is estimated with the estimator given below,

$$
\begin{array}{ll}
\text { Minimize: } & (A \hat{X}-P){ }^{2} \\
\text { subject to: } & B \hat{X} \leq 0 \\
& \hat{X} \geq L B \\
& \hat{X} \leq U B \\
& \hat{X}(i) \in \mathbb{Z}, 1 \leq i \leq N \\
& \hat{X}(N+1) \in \mathbb{R}
\end{array}
$$

as $B, L B$ and $U B$ defined below.

$$
\begin{gathered}
B=\left(\begin{array}{cccccc}
-1 & 1 & 0 & \ldots & 0 & 0 \\
0 & -1 & 1 & \ldots & 0 & 0 \\
: & : & : & : & : & : \\
0 & 0 & \ldots & -1 & 1 & 0 \\
c_{1} & -1 & 0 & \ldots & 0 & 0 \\
0 & c_{2} & -1 & \ldots & 0 & 0 \\
: & : & : & : & : & : \\
0 & 0 & \ldots & c_{N-1} & -1 & 0
\end{array}\right), \\
L B(i)=0,1 \leq i \leq N+1, \\
U B=\left[\frac{T}{\omega_{-N / 2}}, \frac{T}{\omega_{-N / 2+1}}, \ldots, \frac{T}{\omega_{N / 2}}, T\right]^{\prime} .
\end{gathered}
$$

This is a mixed integer programing problem having one real variable $(\hat{\tau})$ and $N$ integer variables $\left(\hat{k_{n}}\right)$. All the constraints are linear, but the cost function is nonlinear. This constrained least squares problem with mixed integer variables delivers a unique solution in $\left[0 T_{S Y M}\right)$ and it is NP-hard.

\subsection{Coarse Step}

As stated in Section 3.1, the solution of the constrained least squares problem is unique in one OFDM symbol period range. Therefore, symbol synchronization has to be achieved before applying the estimator. Symbol synchronization is a well studied subject, in this work the double auto-correlation synchronization method [7] is employed. This method is based on calculating two normalized auto-correlation metrics, $M_{1}$ and $M_{2}$ :

$$
\begin{aligned}
& M_{1}[l]=\frac{\sum_{m=0}^{N_{s}-1} r[l+m] r^{*}\left[l+m+N_{s}\right]}{\sum_{m=0}^{N_{s}-1}|r[l+m]|^{2}}, \\
& M_{2}[l]=\frac{\sum_{m=0}^{N_{s}-1} r[l+m] r^{*}\left[l+m+2 N_{s}\right]}{\sum_{m=0}^{N_{s}-1}|r[l+m]|^{2}} .
\end{aligned}
$$

The difference of these two metrics has a maximum at the beginning of the last correlation window in the sequence. The time of arrival estimation of this step is $\hat{\tau}_{1}=\hat{k} T_{s}$, where $T_{s}$ is the sampling period and $\hat{k}$ is the first sample of the signal estimated in the coarse step.

The range is uniformly distributed in the interval [0Rmax], in the absence of noise so the delay estimation error is uniformly distributed in the interval $\left[-\frac{T_{s}}{2} \frac{T_{s}}{2}\right]$. Therefore, when the noise in the channel is not dominating the estimation performance, the precision of $\hat{\tau_{1}}$ is limited by (21).

$$
\operatorname{var}\left\{\left|\hat{\tau}_{1}-\tau\right|\right\} \geq \frac{T_{s}^{2}}{12}
$$

A parameter sweep for $T$ in the SNR region of interest allows to derive minimum signal duration to ensure correct synchronization. When the estimation error $(\epsilon)$ satisfies (22), the coarse step is locked on the correct symbol.

$$
\left|\tau-\hat{\tau_{1}}\right|=|\epsilon| \leq \frac{T_{S Y M}}{2}
$$

\subsection{Fine Step}

Fine and super fine range estimation steps are based on the frequency domain estimator as stated in Section 3.1. The constrained least squares mixed integer problem is divided into two problems to be solved in these two steps. the first estimation $\hat{\tau_{1}}$ is fine tuned in the fine step, when the coarse step is locked on the correct symbol. $\tau_{2}=\tau-\hat{\tau}_{1}$ is estimated in the fine step to calculate the integer values $k_{n}$. 


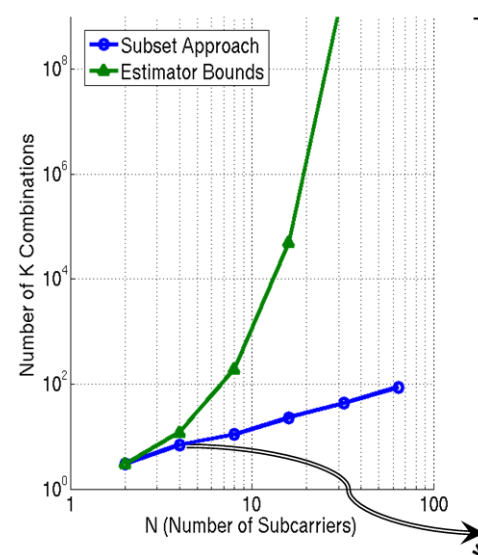

(a)

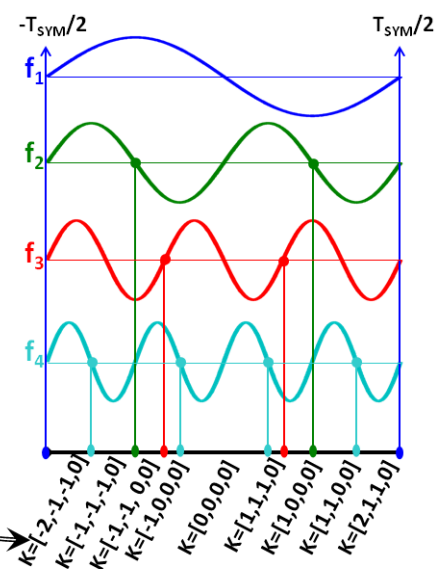

(b)
Fig. 1. Relation between $M$ and $N$ for equidistantly spaced subcarriers, $B=6 \mathrm{GHz}$ and $\epsilon_{\max }=0.5 \mathrm{~ns}$. The $K$ set in this example is formed as $K=\left[k_{4}, k_{3}, k_{2}, k_{1}\right]$.

The absolute phases of the subcarriers are calculated by DFT of the symbol synchronized in the coarse step:

$$
\begin{aligned}
& \Theta_{n}=\omega_{n} \tau_{2}+\omega_{c} \tau=2 \pi k_{n}+\theta_{n}+\omega_{c} \tau \\
& \Theta_{n}, \theta_{n} \in[-\pi / 2 \pi / 2),
\end{aligned}
$$

where phase of the carrier frequency $\left(\omega_{c} \tau\right)$ is common for all absolute phases. The carrier frequency information is removed and the phase relative to $\Theta_{1}$, given in (24), are used. The relative quantities are denoted by a superscript $r$.

$$
\Theta_{n}^{r}=\omega_{n}^{r} \hat{\tau}_{2}=2 \pi k_{n}^{r}+\theta_{n}^{r},
$$

where $\Theta_{n}^{r}=\Theta_{n}-\Theta_{1}$ and $\omega_{n}^{r}=\omega_{n}-\omega_{1}$. Using the relative phases $\Theta_{n}^{r}$, a baseband estimator can be derived to estimate $\tau_{2}$. However, that new estimator will also be a constrained least squares mixed integer problem even with nonlinear constraints on $\hat{k_{n}}$ since $\tau_{2}$ is not necessarily positive. Instead, Algorithm 1 proposed to split one big constrained least squares mixed integer problem into multiple low complex least squares problems. In the algorithm,

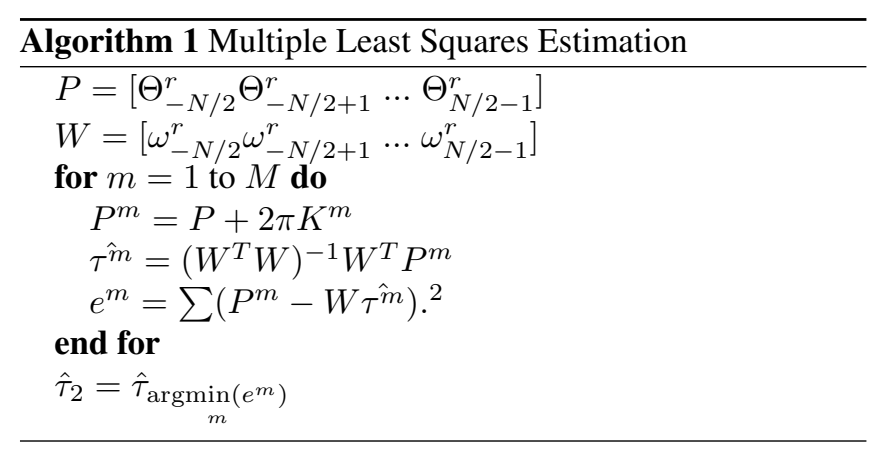

$M$ possible $K^{m}$ vectors are evaluated, given that $K^{m}$ is $\left[k_{-N / 2}^{r} k_{N / 2+1}^{r} \ldots k_{N / 2-1}^{r}\right]^{T}$. For each set $m, \hat{\tau}^{m}$ is estimated

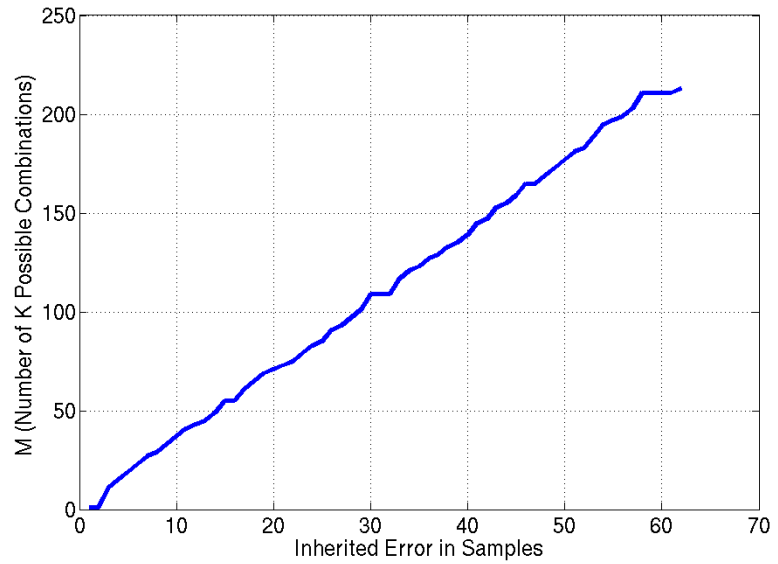

Fig. 2. Relation between $M$ and $\epsilon$ for $N=16, f_{s}=12 \mathrm{GHz}$, $B=6 \mathrm{GHz}$.

by solving the equation set $W \tau^{\hat{m}}=P^{m}$ by linear least squares as given in Algorithm 1. Therefore $M$ least squares problems will have to be solved. Using the bounds in (15) and (16), all $K^{m}$ vectors that can be returned by the estimator are obtained from a search tree with $N$ layers. As shown in Figure 1.a, the total number of $M$ increases exponentially with $N$ such that $M(N)=\mathcal{O}\left(2^{N}\right)$. Therefore, the maximum estimation error $\left(\epsilon_{\max }\right)$ inherited from the coarse step stated in (25) is taken into consideration to find $K^{m}$ set more precisely.

$$
-\frac{T_{S Y M}}{2} \leq-\epsilon_{\max } \leq \hat{\tau}_{2} \leq \epsilon_{\max } \leq \frac{T_{S Y M}}{2}
$$

Given the ambiguity of the coarse step, appropriate $K^{m}$ vectors can be trimmed from the search algorithm. As shown in Figure 2, $M$ linearly increases with the inherited error. The possible combinations are calculated by dividing the complete solution space $\mathbb{S}=\left\{x \mid x \in\left(-\epsilon_{\max }, \epsilon_{\max }\right)\right\}$ into subsets where only one $K^{m}$ is possible. An example for the subset based approach is shown in Figure 1.b for $N=4$. With this approach, $M$ increases linearly with $N$ as shown in Figure 1.a. Even for $N=16$, there is a factor of $10^{4}$ difference between the number of combinations calculated by these two methods which means a factor of $10^{4}$ benefit in computational cost.

Furthermore, the $K^{m}$ set analysis is only done offline, and the sets are recorded to be used in the ranging algorithm. So, this graphical analysis shown in Fig. 1.b is not an overload to the ranging algorithm.

\subsection{Super Fine Step}

The coarse and the fine steps form a baseband estimator together. The baseband estimation $\left(\hat{\tau}_{2}+\hat{\tau}_{1}\right)$ is used to estimate $k_{n}$, which are the integer variables of the constrained 
least squares mixed integer problem. Therefore, the super fine step, which is the second step for solving the constrained least squares mixed integer problem, is simplified to one least squares problem.

The phase of the carrier frequency can be written as (26) and with the previous steps, $k_{c}$ can be estimated from (27).

$$
\begin{gathered}
w_{c} \hat{\tau}=2 \pi \hat{k_{c}}-\hat{\rho_{c}} \\
\hat{k_{c}}=\left\lfloor\frac{\hat{\tau}_{2}+\hat{\tau}_{1}}{2 \pi \omega_{c}}\right\rfloor .
\end{gathered}
$$

In order to estimate $\rho_{c}$, the absolute phases are used, since the integer variables are already estimated by previous steps.

$$
\hat{\rho}_{c}=\operatorname{mean}\left(\bar{\Theta}-W \hat{\tau}_{2}\right),
$$

where, $\bar{\Theta}=\left[\Theta_{-N / 2}, \Theta_{-N / 2+1}, \ldots, \Theta_{N / 2-1}\right]^{\prime}$. Finally the ToA estimation is

$$
\hat{\tau}=\frac{2 \pi \hat{k_{c}}-\hat{\rho_{c}}}{\omega_{c}}
$$

Super fine step is capable of tuning the ToA estimation in $\left[-\frac{1}{2 f_{c}} \frac{1}{2 f_{c}}\right)$, so in order to benefit from this final step, fine estimation error should be lower than $\frac{1}{2 f_{c}}$. Since the additive noise is Gaussian, with $99.7 \%$ confidence level, fine estimation error is smaller than $3 \sigma$. Therefore, to benefit from super fine step, condition (30) should be provided.

$$
\left(\frac{1}{6 f_{c}}\right)^{2} \geq \frac{3}{4 \pi^{2} T S N R\left(\left(f_{c}+\frac{B}{2}\right)^{3}-\left(f_{c}-\frac{B}{2}\right)^{3}\right)}
$$

\subsection{Signal Design}

The complete signal and its parts are given in Figure 3. STS, short training symbol, is one OFDM symbol defined in (1). Its duration is equal to the period of the OFDM symbol, $T_{S Y M}$.

Multiple STSs form a Correlation window, CW, such that length of one $\mathbf{C W}\left(T_{C W}\right)$ is longer than the maximum delay $\frac{R \max }{c}$, in order to prevent ambiguity to detect false synchronization points inside the range of interest:

$$
T_{C W}=N_{S T S} \times T_{S Y M}>\frac{R \max }{c}
$$

For the double auto-correlation method, there should be a sudden change in the signal after at least 3 CWs. Therefore, the synchronization sequence, $\mathbf{S S}$, is formed by three negative CWs followed by three positive ones. Overall signal contains multiple copies of the same sequence: SS. The copies are used for averaging, total length of the signal is determined considering the typical received SNR for the application.

Moreover, the total number of STSs $\left(N_{S T S}^{*}\right)$ to be used for frequency domain analysis should be

$$
N_{S T S}^{*} \geq \frac{3 /\left(6 f_{c}\right)^{2}}{4 \pi^{2} T_{S Y M} S N R\left(\left(f_{c}+\frac{B}{2}\right)^{3}-\left(f_{c}-\frac{B}{2}\right)^{3}\right)} .
$$

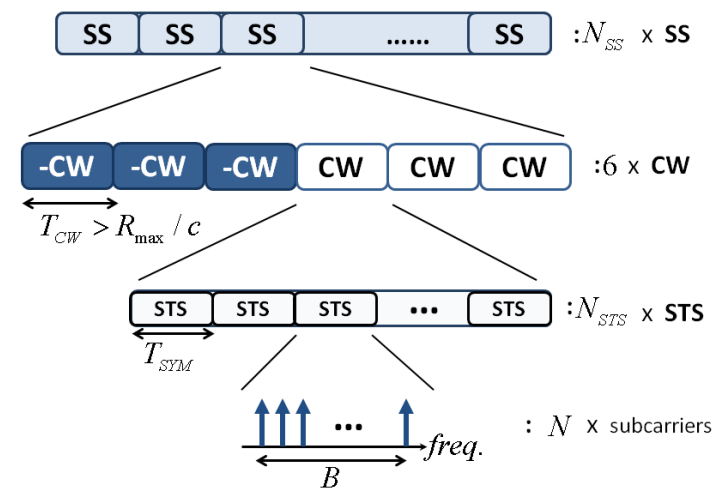

Fig. 3. Signal construction. The smallest unit of the signal STS is used to construct the signal.

\section{SIMULATIONS}

Assuming a state of the art power amplifier for $60 \mathrm{GHz}$ has a $15 \mathrm{dBm}$ output power in the linear region [8], the receiver has $10 \mathrm{~dB}$ noise figure [9] and omnidirectional antennas are used, the typical value of received SNR at $20 \mathrm{~m}$ is around $0 \mathrm{~dB}$. Therefore, simulation setup is designed for achieving $0.1 \mathrm{~mm}$ precision below $0 \mathrm{~dB}$.

Simulation result for a signal that does not ensure the condition (32) is plotted in Figure 4 to show the timing relations between the steps. In the Figure, the performance of the coarse step had reached its limit at $0 \mathrm{~dB}$ and is not changing with increasing the SNR. That means, the highest precision determined by sampling frequency is obtained from this step. From the point that the first stage locks on the correct symbol, the estimation error in the baseband estimator starts to decrease with the Cramér-Rao lower bound drawn for $f_{c}=0$. The estimation error of the super fine step can follow the bound only after the precision of the second step is better than $1 /\left(6 f_{c}\right)$ as shown in (30). Before that point, estimation error of fine and super fine steps follow the same curve.

Using the proposed implementation-aware estimator to its full force by ensuring (32) and correct synchronization in coarse step, a precision of $0.1 \mathrm{~mm}$ is obtained at $0 \mathrm{~dB}$ received SNR as shown in Figure 5. The performance of the estimator approaches the CRLB without assuming infeasible resolution and within acceptable computational complexity.

\section{CONCLUSION}

A ToA estimator to be used in ranging application is derived based on frequency domain representation of the transmitted signal. In order to increase its solution space to meet the maximum range, it is combined with symbol synchronization in a coarse estimation step. The subsequent finer estimation results in an NP-hard constrained least squares mixed integer problem. For implementation reasons it is solved in two steps: 


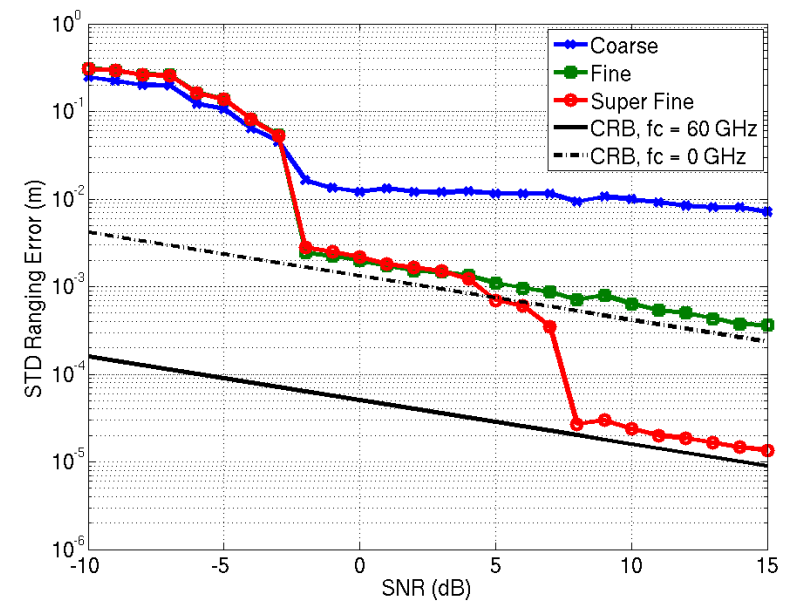

Fig. 4. Understanding the relations between steps. 20 randomly selected range values between $1 \mathrm{~cm}$ to $18 \mathrm{~m}$ are simulated. $N=16, N_{S S}=20, N_{S T S}^{*}=10 B=6 \mathrm{GHz}$, $f_{c}=60 \mathrm{GHz}$, total duration of the signal is $7.2 \mu \mathrm{s}$. Each simulation is repeated for 500 times.

fine and super fine steps. Carefully selecting the least squares problems to be solved in this step highly reduced the computational cost and built a link between number of least squares problems and the performance of the coarse step. This relation between coarse and fine steps provide flexibility against implementation trade-offs between transmitted signal energy, computational cost and ranging precision. Stepwise approach also brings flexibility for future improvements such as channel and clock offset equalization, so this estimator can be strengthen against realization and environment imperfections, and it is suitable for real world implementation.

The performance of the estimator is evaluated for a single path channel with AWG noise and a precision under $1 \mathrm{~mm}$ is obtained for received SNR lower than $0 \mathrm{~dB}$. To the authors' knowledge, this result is the first efficiently implementable estimator achieving the precision to the CRLB for sub-mm precision.

\section{REFERENCES}

[1] N. Van Helleputte, M. Verhelst, W. Dehaene, and G. Gielen, "A reconfigurable, $130 \mathrm{~nm}$ cmos $108 \mathrm{pj} /$ pulse, fully integrated IR-UWB receiver for communication and precise ranging," Solid-State Circuits, IEEE Journal of, vol. 45 , no. 1, pp. $69-83$, jan. 2010.

[2] C. Zhang, M.J. Kuhn, B.C. Merkl, A.E. Fathy, and M.R. Mahfouz, "Real-time noncoherent uwb positioning radar with millimeter range accuracy: Theory and experiment," Microwave Theory and Techniques, IEEE Transactions on, vol. 58, no. 1, pp. 9 -20, jan. 2010.

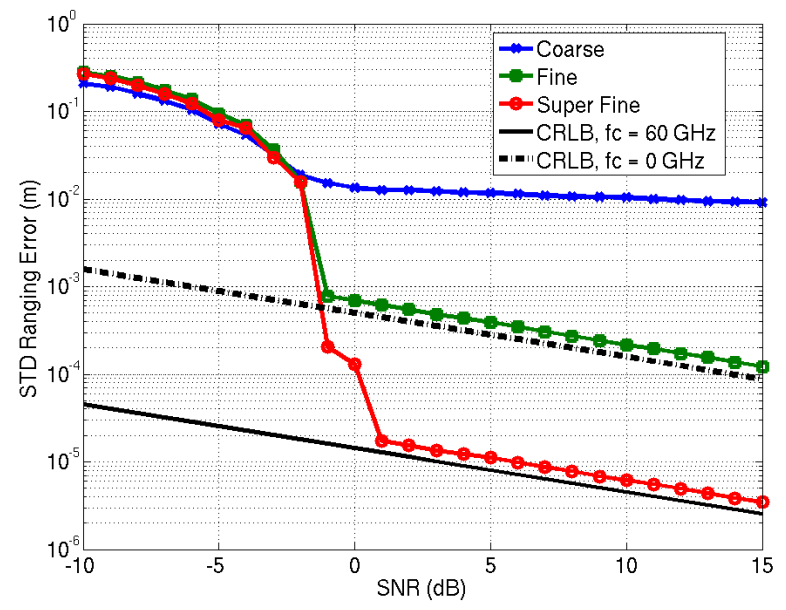

Fig. 5. Simulation Results for 20 randomly selected range values between $1 \mathrm{~cm}$ to $18 \mathrm{~m} . N=16, N_{S S}=25, N_{S T S}^{*}=$ $100 B=6 \mathrm{GHz}, f_{c}=60 \mathrm{GHz}$. Each simulation is repeated for 500 times. total duration of the signal is $9 \mu \mathrm{s}$.

[3] C. Meier, A. Terzis, and S. Lindenmeier, "A robust 3D high precision radio location system," in Microwave Symposium, 2007. IEEE/MTT-S International, june 2007, pp. $397-400$.

[4] Z. Sahinoglu, S. Gezici, and I. Guvenc, Ultra-Wideband Positioning Systems, Cambridge University Press, 2008.

[5] T. Redant and W. Dehaene, "High resolution time-ofarrival for a cm-precise super 10 meter 802.15.3c-based 60 ghz OFDM positioning application," in 2nd International Conference on Pervasive and Embedded Computing and Communication Systems (PECCS 2012), feb. 2012, vol. 2, pp. $217-277$.

[6] A. Quazi, "An overview on the time delay estimate in active and passive systems for target localization," Acoustics, Speech and Signal Processing, IEEE Transactions on, vol. 29, no. 3, pp. 527 - 533, jun 1981.

[7] K. Wang, M. Faulkner, and I Tolochko, "Timing synchronization for 802.11a WLANs under multipath channels," in ATNAC 2003, 2003.

[8] Jiashu Chen and A.M. Niknejad, "A compact 1v 18.6dbm $60 \mathrm{ghz}$ power amplifier in $65 \mathrm{~nm}$ cmos," in Solid-State Circuits Conference Digest of Technical Papers (ISSCC), 2011 IEEE International, feb. 2011, pp. 432 -433.

[9] F. Giannetti, M. Luise, and R. Reggiannini, "Mobile and personal communications in the $60 \mathrm{ghz}$ band: A survey," Wireless Personal Communications, vol. 10, pp. 207-243, 1999. 